\title{
AN INVESTIGATION OF FRICTIONAL AND COLLISIONAL POWDER FLOWS USING A UNIFIED CONSTITUTIVE EQUATION
}

\author{
M. Kheiripour Langroudi ${ }^{1}$, S. Turek ${ }^{2}$, A. Ouazzi ${ }^{2}$ and G. I. $\operatorname{Tardos}^{1}$ \\ 1- The City College of the City University of New York, USA \\ Tardos@CCNY.CUNY.Edu, mkheiripour@gc.cuny.edu \\ 2- Institute of Applied Mathematics, TU Dortmund, Germany \\ ture@featflow.de, ouazzi@math.uni-dortmund.de
}

\begin{abstract}
This is an experimental and numerical study of dry, frictional powder flows in the quasi-static and intermediate regimes using the simple geometry of the Couette device. We measure normal and shear stresses on the shearing surface and propose a constitutive equation valid in both the slow frictional, quasi-static and the intermediate (dense) collisional regimes of flow. This constitutive equation is then used in a new, specially developed numerical scheme to solve the continuum equations of motion and to obtain stress and velocity distributions in the powder. While the measurements to obtain the constitutive equation are performed in a concentric Couette device, the numerical scheme is used to predict the torque and stresses in two additional geometries: an eccentric Couette device where the inner, rotating cylinder is placed off-center with different eccentricities and a more complicated geometry where a cylindrical body is introduced in the middle between the rotating and stationary cylinders and obstructs part of the shearing gap. Further experiments are then conducted in the two new geometries and the torque on the inner, rotating cylinder is measured and compared to the numerical solution.
\end{abstract}

We find experimentally, that it is possible to measure normal stresses on the shearing wall of the Couette device inside the granular layer and calculate the ratio of the average shear to normal stress as a function of shear rate. It appears that the powder's dynamic angle of friction is reproduced by this ratio only at very low shear rates. As the shearing rate increases, the ratio of the stresses also increases due to collisions between particles that sustain loads in addition to dry friction that is prevalent at low shear rates. We show that a modified Couette device with slow axial flow superimposed on the shearing motion induced by the rotating cylinder can be used to determine the constants ("b" and " $\mathrm{n}$ ") of a yield condition for any powdery material that is somewhat free flowing. The yield condition is valid in both the quasi-static as well as the "intermediate" regime of flow and contains a term characterizing "solid"-like behavior and an additional term that captures some "fluid"-like properties at higher shear rates.

The paper also describes a new finite element solution, realized in the FEM solver FleatFlow, of the generalized Navier-Stokes equations that uses, in addition to the yield condition determined above, a generalized viscosity that describes a Newtonian fluid, a Bingham Plastic, an incompressible frictional powder (Schaeffer solid) and a power-law fluid. We use the numerical method to validate some experimental measurements and calculate the torque in the Couette device in three different geometries: a concentric, two cylinder, arrangement and two new geometries in which the cylinder is positioned eccentric in the 
Couette and one where an additional cylindrical object is placed into the shearing gap and obstructs parts of it.

\section{A. Experimental Program}

Powder flows have been studied extensively in the past starting with the pioneering work of Jenike (1954) and Jackson (1986). The accent of this earlier work was to establish the condition under which a stressed layer of powder will rupture and the material will start to flow. More recent studies were dedicated to modeling powder flows using mostly numerical methods of various kinds: Luding (2005), Moreno-Atanasio et al., (2005), Muguruma et al., (2000), Srivastava and Sundaresan, (2003), just to mention a few. Extensive work by Savage (Savage, 1998) and Tardos and co-workers (Tardos et al., 1998 Tardos et al., 2003 and Tardos and Mort, 2005) looked into the slow and intermediate regimes of flow at slow and moderately high shear rates and the transition from one to another. The study of continuum models to describe powder flows was pioneered by Schaeffer, (1987) and continued by Tardos, (1997) and Quazzi et al., (2005), among others. In the present study we explore experimentally and numerically the transition of powder flows from slow to moderately fast by using a Couette device.

\section{Background - batch and continuous-flow Couette device}

A schematic representation of the Couette device used during the present investigation is shown in Figure 1 with details of the sensors and the rotating cylinder given on the RHS of the figure. The vertical shear gap forms between the rotating and the stationary cylinders and both its width and height can be adjusted by appropriate choice of the radius and height of the rotating cylinder. The material is fed from above using a vibratory feeder (not shown in the figure) and is discharged by a screw-in-cylinder metering device (also not shown) that transfers the material to a precision balance for flow rate measurement. The walls of the Couette are made rough by gluing sand paper on the shearing surfaces as shown in the figure. The roughness of the walls is chosen to match or exceed the coefficient of internal friction of the material thereby trying to assure a non-slip boundary condition. By closing the discharge section, the Couette device can be operated in batch mode or, by feeding and removing material to achieve a steady state vertical flow one can operate the device continuously. The material above the rotating cylinder (denoted overburden in the figure) is stationary and only provides dead weight to the shearing layer.

Experiments in the Couette device were performed without (batch) and with axial flow (continuous), and several depths overburdens to control the pressure in the shearing gap. Normal stresses were measured on both the outer, stationary wall as well as the inner, rotating (shearing) wall of the device as shown in the figure. Shear stresses where measured indirectly and recalculated from the torque on the rotating cylinder.

A typical batch, filling curve of the Couette is shown in Figure 2 for $1 \mathrm{~mm}$ in diameter round glass beads with a cylinder height of 20 inches and diameter of 1.5 inches, in a housing of six inches in diameter (with a gap of 2 and $1 / 4$ inches). We distinguish between the increasing portion of the torque as a function of height (LHS of the figure) and the torque due to packing of the material at constant height and increasing time (RHS of the figure). First, we concentrate on the LHS region where the torque is height dependent as the Couette is filled with particles and fit the equation of the torque, $T$ to the measured data as was done previously by Tardos et al., [1998]: 


$$
T=(\tau R) 2 \pi R h=\pi \rho_{B} g h^{2} R^{2} \sin (\phi)
$$

where $\tau=p \sin \phi$ is the shear stress and $p$ is the average normal stress given by $\mathrm{p}=\rho_{\mathrm{B}} \mathrm{gh} / 2$. Other notations in equation (1) are: $\rho_{\mathrm{B}}$ is the bulk density, $\mathrm{g}$ is the acceleration of gravity, $\mathrm{h}$ is the height of material in the vertical direction (function of filling time, see figure $1 b.), R$ is the cylinder radius and $\phi$ is the angle of internal friction of the material. The implication of equation (1) is that the shear stress $\tau$ is linearly dependent on the height $h$ so that when the torque is calculated the height appears to the second power. In addition, the theoretical line fits the experimental results quite well suggesting that the shear stress is indeed a linear function of height as long as the material is continuously fed to the system. These general characteristics of the device were already established in our earlier work mentioned above.

We now concentrate on the second part of the curve where the torque increases due to packing of the material at constant height. Additional experiments were performed with a larger inner cylinder of 4 inches in diameter so that the shearing gap was approximately one inch and three different curves of the same kind are reproduced in Figure 3a for small (0.1 $\mathrm{mm})$, medium $(0.5 \mathrm{~mm})$ and large $(1.0 \mathrm{~mm})$ diameter glass particles. One can easily see the different rates at which these particles pack at a constant shearing rate (15 RPM of the rotating cylinder): larger particles pack at a much higher rate due to their enhanced capability of bridging the relatively small shear gap. It appears from Figure $3 a$ that the trends shown would continue but in reality, the range of the torque-meter is exceeded and the experiment has to be stopped.

Figure $3 \mathrm{~b}$ depicts similar behavior for the $0.1 \mathrm{~mm}$ in diameter glass beads but for a much wider shear gap ( 2 and $1 / 4$ inches). Here, we calculated the average shear stress using the equation

$$
\tau_{\text {ave }}=T / 2 \pi R^{2} L
$$

As seen in the figure, the torque increases significantly during the packing phase but does not exceed the range of the torque-measuring device. This is mainly due to the shorter cylinder used and by the relatively large shear gap that allows some additional movement of the granular layer in the radial direction. It appears from the figure that the torque has reached its steady state value at the lowest shear rate (blue curve) and we use this experiment to show how the shear stress depends on shear rate when the Couette is operated in batch mode (without vertical flow) at constant height. As seen in Figure 3b, increasing the shear rate more then five-fold does not result in any significant change in the shear stress: the material is in the frictional, Quasi-static regime and the average shear stress is practically independent of shearing rate.

We reproduced in Figure 4 a similar experiment to that shown in Figure $3 \mathrm{~b}$ but allowing material to flow at a small rate vertically in the shear gap. The torque in this case decreases significantly due to a minute change in solid fraction (porosity) that allows chains of particles to break as the inner cylinder rotates. Using equation (2), the average shear stress can be calculated as a function of shear rate as shown in Figure 4. As seen, the shear stress exhibits two very distinct regimes: a quasi-static regime where the shear stress is mostly independent of the shear rate (at low shear rates) and an additional regime ("intermediate" 
as denoted in the figure) where the dependence takes the form of an increasing function. We assume that the behavior resembles a power-law with an index $n=0.5-1$. In fact, the power-law index appears to be variable, increasing at higher shear rates. One would assume that continuing to increase the shear rate would yield even higher values of the index " $\mathrm{n}$ " as the flow approaches the inertial regime (where the coefficient should be $n=2$ ) but such experiments are outside the capability of our Couette device since centrifugal forces take over and the material is pushed radially outward and a slip condition sets in near the rotating cylinder.

The purpose of these experiments was to show the significant difference in behavior of the shearing layer in the batch, no-flow and the continuous-flow device. While in the batch mode, the material continues to pack increasing its solid fraction as a function of time, in the flowing system a steady state sets in at an approximately constant solid fraction. This solid fraction is beyond a certain critical value, somewhat characteristic for each powder, where the material can "dilate" sufficiently and "flow" under shear. In this expanded, mobilized (sometimes also called "fluidized", even though there is no gas present to actually fluidize the powder) condition, the powder can transit from the quasi-static to the intermediate regime as the shearing rate is increased. We showed in separate experiments that the influence of the vertical flow rate is negligible if it can be kept small, just enough to prevent packing.

The remainder of the paper is concerned with this second case where there is a minute flow of material in the vertical direction and where a quasi-static, shear-rate-independent and an intermediate, dense-phase, shear-rate-dependent shear stress (torque) develops. The main goal of the experiments is to measure the shear and normal stresses on the shearing wall, their location and their ratio. We further propose a yield condition and a constitutive equation (closure) derived entirely from experimental measurements and develop a new numerical scheme to solve the momentum equations with the newly proposed closure, to cover both regimes of flow.

\section{Stress measurements in the continuous-flow Couette device}

Experiments were performed to show the dependence of the shear and normal stresses on the shear rate. We use as test material glass beads and chips of different size and shape.

\section{a. Average shear stress measurements and their location.}

Since there are no direct shear-stress measuring sensors commercially available that can be employed in a dense granular bed, the shear stress on the rotating cylinder was measured indirectly using the torque on the shaft exerted by the granular medium on the entire length, $L$ of the cylinder as in equation (2). To explore the dependence of the shear stress on the depth of the granular layer, we employed cylinders of different length, $L=4,8,12$ and 16 inches (and diameter of 4 inches) and calculated the average shear stress in each case. The experiments were performed with no overburden (material on top of the rotating cylinder, see Figure 1) using $0.5 \mathrm{~mm}$ in diameter glass beads and an axial flow rate of $1 \mathrm{~cm}^{3} / \mathrm{sec}$. Results are given in Figure 5 where the average shear stress is given as a function of cylinder height, $L$ for different shear rates by rotating the inner cylinder from 5-45 RPM. As seen, the shear stress is approximately linear as a function of height and depends strongly on shear rate as already shown above. 
Another interesting observation is that, by forcing the trend of the experimental measurements to go to zero stress at zero height, data from the shortest cylinder ( $L=4$ inches in length) fall above the line at a somewhat higher shear stress. This behavior is due to friction between the lower cover of the cylinder and the granular material (in addition to friction in the shearing layer between the cylinders) which is not a linear function of height; this result was already demonstrated by DaCruz et al., (2005). When the length, L (height) of the shearing layer is small, the influence of this portion of the cylinder is significant and hence the torque is higher. At larger length (height), the influence of the bottom cover becomes less significant and the dependence is almost perfectly linear. The main conclusion from this measurement is that using cylinders longer (taller) then about 8 inches will mostly eliminate the influence of the end effect (lower cover) and that the value of the average shear stress will most likely occur at the middle point over the length, $L$.

\section{b. Normal stress measurements.}

Figure 6 shows a schematic representation and a picture of the instrumentation to measure normal stresses inside the bulk material on the rotating cylinder at three depths $\left(l_{1}, l_{2}\right.$ and $\left.l_{3}\right)$. The stress sensor is commercially available from Tekscan, Inc. (Boston Ma) and is made of a circular sensitive element $1.1 \mathrm{~cm}$ in diameter glued to the surface of the rotating cylinder and an electronic circuit situated inside the blue box in the picture. Since the cylinder is rotating inside the granular layer, the signal cannot be hard-wired but is instead transmitted by radio to a receiver connected directly to the data acquisition system. To our knowledge, this is the first time that such a measurement was undertaken on a shearing surface inside a granular medium. The cylinder used is 4 inches in diameter (this dimension is imposed by the size of the radio-transmitter) and 20 inches long (to accommodate the three radio transmitters). The outside diameter of the stationary cylinder that forms the Couette device (not shown in the figure) is 6 inches in diameter and thus defines a granular layer of one inch in thickness that surrounds the sensors.

These particular experiments were performed with an overburden of approximately four inches so that the depth of the three sensors was $l_{1}-l_{3}$ plus the overburden as shown in Figure 7. Different rotation rates from 5-60 RPM and glass beads of $0.5 \mathrm{~mm}$ in diameter were used. The experiment was performed with an overall vertical flow-rate of approximately $2 \mathrm{~cm}^{3} / \mathrm{sec}$; this results in a very slow axial velocity in the Couette gap of less then 0.1 $\mathrm{mm} / \mathrm{sec}$ and does not influence significantly the flow and stresses in the radial direction.

Figure 7 depicts the results of the stress measurements as a function of depth. The distribution appears to be linear with the error bars representing the fluctuation of stress and the dependence on the applied shear. As seen, the normal stress in the sheared layer is not shear rate dependent even at the highest shear rate (at 60 RPM), within the error of the measurement and the presence of fluctuations. This allows the calculation of an average normal stress on the rotating cylinder that appears, according to these results, to reside in the middle of the cylinder (L/2). This is an important conclusion since it shows that both the average shear and normal stresses are practically located around the midpoint of the rotating cylinder at least under the experimental conditions of this experiment. In subsequent experiments only one normal sensor, situated at the midpoint on the cylinder, was used. 


\section{c. Ratio of Shear to Normal stresses in the Couette device.}

The most interesting result from the above measurements is that one can calculate the ratio of the average shear to normal stresses as a function of shear rate. A typical result is shown in Figure 8 where the average shear stress and local normal stress measured in the middle of the rotating cylinder (L/2) are shown as a function of shear rate. The particles used are 1 $\mathrm{mm}$ in diameter spherical glass beads. The shear stress has the overall behavior already described in Figure 4 as the stress is practically constant at low shear rates and increases significantly as soon as the material reaches the intermediate regime of flow. The normal stress shows a very interesting behavior: it is constant and lower at low shear rates (where the shear stress is also constant) and then increases abruptly and remains practically constant thereafter even though the shear stress increases continuously.

The ratio of the two stresses (apparent friction coefficient) is given in Figure 9 and, as seen, the ratio exhibits a similarly interesting behavior: at very low shear rates, the ratio is constant and only slightly lower then the tangent of the friction coefficient of glass particles (about 0.5) as one would expect from quasi-static flow theory. As the dimensionless shear rate increases beyond a certain critical value (in this case about $1.0 \mathrm{sec}^{-1}$.), the ratio of shear stress vs. normal stress increases significantly. This behavior is mainly due to the superposition of collisions between particles on the sliding friction of surfaces so that the overall shear stress and "friction coefficient" also increase. This kind of behavior was already suggested by simulations (Campbell, 2006 and DaCruz et al., 2005) but never actually measured experimentally before. It also shows that the material moves from the quasi-static regime to the intermediate regime of flow where collisions become more important and actually carry some load. A power-law type curve is fitted to the data: it shows a power-law coefficient of $n=0.72$ and a constant coefficient $a=0.39$ for zero shear rate. In the same figure we show for comparison an equivalent correlation for crushed, odd-shaped glass particles of nominal size of $1 \mathrm{~mm}$. As seen, the power-law index is somewhat higher, as expected from considerations of collisional impacts between non-spherical particles $(n=1.03)$ and a higher constant coefficient $(a=0.48)$ due to the higher internal angle of friction.

We conclude from this work that it is possible to measure normal stresses on the sharing wall of the Couette device inside the granular layer and calculate the ratio of the average shear to normal stress as a function of shear rate. It appears, as suggested by previous theoretical simulations, that the dynamic angle of friction is reproduced by this ratio only at very low shear rates. As the shearing rate increases, the ratio of the stresses also increases due to collisions between particles that sustain loads in addition to dry friction that is prevalent at low shear rates. Our experiment could not probe the higher limit of the "friction coefficient" in the rapid granular flow regime (when only collisions are important) because of equipment limitations and the inherent presence of gravity that makes at least some enduring contacts between particles unavoidable.

\section{Proposed yield condition and constitutive equation}

We try to generalize the above findings for the case when the powder transitions from the quasi-static to the intermediate regime of flow and propose a yield condition that holds at low and higher shear rates. We show below how the experimental yield condition obtained from experiments in the Couette device and given in Figure 9 can be inserted into the Schaeffer constitutive law (Schaeffer, 1987). From Figure 9, one can fit the following equation to the data: 


$$
\frac{\tau}{\sigma}=a+\left.b|\cdot|\right|^{n}
$$

where $\tau$ and $\sigma$ are the shear and normal stresses and "a", "b" and "n" are coefficients characterized by the experimental curve. We use the notation for the modulus of the shear rate $|\dot{\gamma}|$ to designate that only its magnitude is of relevance and not its direction. A slight generalization can be obtained by replacing the constant coefficient "a" by tan $\phi$ to obtain:

$$
\frac{\tau}{\sigma}=\tan (\phi)+b \mid \dot{\gamma}^{n}
$$

This equation has the advantage that it reduces to the Coulomb yield condition at zero shearing rate. The error between equations (3) and (4) comes from the complex way the two stresses are measured using independent instrumentation and calibration procedures for the torque and normal stress, respectively.

An equivalent representation of the Coulomb yield condition $\tau=\sigma \tan \phi+c$ can be obtained from the characteristic Mohr circle, by replacing $\sigma$ by $p$ and $\tau / \tan \phi$ by $q / \sin \phi$, in the form (Nedderman, 1992):

$$
q=p \sin \phi+c \cos \phi
$$

where $q$ is half of the difference between the principal stresses and " $c$ " is referred to as "cohesion".

The deviatoric part of the Schaffer law for flow of a dry powder in the quasi-static regime (Schaeffer, 1987) is

$$
\tau_{i j}=\frac{\sqrt{2} p \sin \phi}{|\dot{\gamma}|} \dot{\gamma}=\frac{\sqrt{2} q}{\sqrt{z}} \dot{\gamma}
$$

where $\dot{\gamma}=\frac{1}{2}\left(\nabla u+(\nabla u)^{T}\right)$ is the rate of deformation tensor, $|\dot{\gamma}|$ is its magnitude and $z$ is twice the second invariant defined as $z=2 \gamma_{I I}=\dot{\gamma}: \dot{\gamma}=|\dot{\gamma}|^{2}=\operatorname{tr}\left[(\dot{\gamma})^{2}\right]$. One recognizes the Coulomb yield condition (5) (with $c=0$ ) in the numerator of equation (6). We replace " $q$ " from equation (5) in (6) with $c=b|\dot{\gamma}|^{n}$, to obtain

$$
\tau_{i j}=\sqrt{2} p\left\{\sin \phi+b \cos \phi|\dot{\gamma}|^{n}\right\} \frac{\dot{\gamma}}{|\dot{\gamma}|}
$$

or in terms of the variable "z":

$$
\tau_{i j}=\sqrt{2} p\left(\sin \phi z^{-\frac{1}{2}}+b \cos \phi z^{\frac{n-1}{2}}\right) \dot{\gamma}
$$

This is the constitutive equation for the modified Schaeffer's law that includes the behavior at higher shear rates, characteristic of the "intermediate" regime of powder flow. The first term 
on the right hand side of eqs. (7) corresponds to the plastic deformation (frictional or solidlike behavior) while the other term corresponds to the viscous behavior (liquid-like) of the granular material. In simple shear flow, in Cartesian coordinates (small gap) where $\mathrm{u}=\mathrm{u}_{\mathrm{y}}$ and $\mathrm{v}=0$, eq. (7) takes the simple form $\tau_{y x}=p \sin \phi\left(1+b \cot a n \phi|\dot{\gamma}|^{n}\right)$.

From a practical point of view, a modified Couette device with slow axial flow superimposed on the shearing motion induced by the rotating cylinder, in which the materials can freely dilate and collide, can be used to determine the values of " $b$ " and " $n$ " for any material that is somewhat free flowing. These values can then be used in equations (7).

\section{B. Continuum theoretical approach}

The purpose of the simulations reproduced below was to predict theoretically experimental results obtained in this work using the simple geometry of the concentric Couette device. Based on these calculations we then predict results for more complex geometries, namely an eccentric Couette and one that has a cylindrical obstacle in the shearing zone. Further experiments are then conducted to check the numerical results.

\section{Equations of motion}

The underlying simulations were performed using a new numerical technique developed specifically to treat flow problems with general constitutive laws for non-Newtonian fluids (see Ouazzi et al. (2005)). The powder is assumed to be an incompressible continuum that obeys conservation of mass:

$$
\frac{D \rho}{D t}=\frac{\partial \rho}{\partial t}+\nabla \cdot(\rho u)=0
$$

where $D * / D t$ is the material derivative and $u$ is the velocity vector. Since we assume that the bulk density $\rho$ is a constant, the continuity equation reduces to the divergence of the velocity $\nabla \cdot u=0$. The powder also obeys a conservation of momentum equation:

$$
\rho \frac{D u}{D t}=-\nabla \cdot T+\rho g
$$

where the stress tensor is given by $T_{i j}=\tau_{i j}-p I$ and where $I$ is the unit tensor. To complete the problem, a closure is required in the form of a constitutive equation that correlates the deviatoric part of the stress tensor $\tau_{i j}$ with the velocity.

The above problem can be similarly formulated in the framework of the generalized incompressible Navier-Stokes equations valid for non-Newtonian fluids and powders:

$$
\rho \frac{D u}{D t}=-\nabla p+\nabla \cdot\left(\mu\left(\gamma_{I I}, p\right) \dot{\gamma}\right)+\rho g, \quad \nabla \cdot u=0
$$

where we take $\tau_{i j}=\mu\left(\gamma_{I I}, p\right) \dot{\gamma}$. To complete the problem, the nonlinear pseudo-viscosity $\mu\left(\gamma_{I I}, p\right)$ is defined as a function of the second invariant of the rate of deformation, or the variable " $z$ ", and the normal stress " $p$ ":

1. Newtonian fluid, $\mu(z, p)=2 \mu_{0}$, where $\mu_{0}$ is the fluid viscosity, 
2. Bingham solid $\mu(z, p)=2 \mu_{0}+\sqrt{2} \tau_{0} z^{-\frac{1}{2}}$, where $\tau_{0}$ is the yield strength,

3. Powder in the quasi-static regime (Schaeffer's law) $\mu(z, p)=\sqrt{2} p \sin \phi z^{-\frac{1}{2}}$,

4. Power-law fluid $\mu(z, p)=2 \mu_{0} z^{\frac{n-1}{2}}$, where " $\mathrm{n}$ " is the power law index,

5. Modified Schaeffer law (from equation (7)) $\mu(z, p)=\sqrt{2} p\left[\sin \phi z^{-\frac{1}{2}}+b \cos \phi z^{\frac{n-1}{2}}\right]$.

A comparison of equation (10) with the classical Navier-Stokes equations (obtained by using eq. (11-1)) reveals that the ordinary viscous terms (proportional to the viscosity $\mu_{0}$ ) have been replaced by shear-rate independent terms (equations (11-2) to (11-5)) that contain the magnitude of the shearing rate $(1 / \sqrt{z})$ in the denominator. This means that these equations are mathematically more complex than the Navier-Stokes equations and apply only when the material is deforming everywhere.

The main mathematical problems of the generalized incompressible continuum material model (Eq. (10)) can be summarized as follows:

- Mathematical analysis: There is a lack of research concerning the existence of solutions for the flow of such "fluids" except for special cases (Hron et al. (2003)), furthermore the dynamic equations (10) show some instability (Bulicky et al. (2008), Bulicky et al. (2005), Prasad and Rajagopal (2006), Malék et al. (2002), Renardy (2003), Schaeffer (1987) and Schaeffer (1990)).

- Singular viscosity: The part of the stress tensor containing $(1 / \sqrt{z})$ is well defined only for non zero values of the rate of strain tensor and for 'non-negative' pressures, which requires some stabilization techniques of singular phenomena due to the nonlinear viscosity (Ouazzi, et al. (2005)).

- Discretization method: It is well known that the computation of solutions to such incompressible systems requires that some care is taken in the choice of the approximating spaces in order to make the discrete problem well posed. Moreover, since a large number of successful spaces satisfying the above condition are nonconforming that present a locking phenomenon for problems involving the rate of deformation tensor, some consistent stabilizing term is required (Turek and Ouazzi (2007)).

- Nonlinear multigrid solver: For this highly nonlinear problem, coupling the pressure and the velocity, there is almost no alternative to linearization using Newton's techniques and therefore efficient multigrid methods for these new types of saddlepoint problems need to be developed (Turek (1998), Turek et al. (2002)).

\section{Finite element method}

The underlying finite element technique, which is the basis for the developed software package FeatFlow, is described in detail in Ouazzi et al. (2005) and a short summary is given in the Appendix. The essence of the special techniques for this kind of flow problems is the introduction of a stabilization term in the denominator that contains the magnitude of the shear stress and a modified multigrid solver. The method was used successfully to solve for incompressible granular flow in a two dimensional hopper using the Schaeffer closure (eq. (11-3)). In the present work we use the same method to solve the modified Navier- 
Stokes equations (eq. (10)) with the new closure that applies for the quasi-static and the intermediate regime (eq. (11-5)) in the Couette geometry and also compare this solution to results obtained for all other closures (eqs. (11)).

The numerical method yields as a first result the velocity and pressure distribution in the gap of the Couette device. Stresses are then computed from the velocity gradients using the constitutive laws (eq. (11-1) to (11-5)) for the respective material. From these results, and $T_{i j}=\tau_{i j}-p I$, the force $(F)$ and torque $(\mathrm{M})$ on the inner, rotating cylinder are calculated as follows:

$$
F=-\oint_{S} T_{i j} \vec{n} d s \quad \text { and } \quad M=-\oint_{S}\left(X-X_{0}\right) T_{i j} \vec{n} d s
$$

In the above equation, $S$ and $X_{0}$ are the surface and the centre of the inner cylinder and $\vec{n}$, is the direction vector.

\section{Computer simulations}

This section is intended to validate the proposed model via the FEM code FeatFlow for complex flow problems (see Appendix). The 2D simulation was done for two concentric cylinders. The outer cylinder, of radius $r_{\text {outer }}$, was kept fixed while the inner cylinder, of radius $r_{\text {inner }}$, was rotated (with $r_{\text {inner }} / r_{\text {outer }}$ set to $2 / 3$ ). The torque was calculated for a section of the cylinders with length unity. All simulations were carried out with $\mu_{0}$ set to unity, the yield strength for the Bingham model $\tau_{0}$ as well as $\sin \phi$ for the Schaeffer solid were set to 0.48 which corresponds to a constant " $a=0.48$ " for crushed glass. The simulations were carried out on a Linux PC with the characteristics shown in Table 1.

\section{Table 1: Linux machine}

Architecture:

CPU type:

CPU frequency:

Number of CPUs:

Main memory:

Linpack test:
Linux 64 2.6.18.8-0.10-default

AMD Opteron 250

$2393 \mathrm{Mhz}$

2 CPUs

$3965 \mathrm{MB}$

3,917 MFLOP/s per CPU

Figure 10 depicts a sample calculation with the numerical method, for four continua including a Newtonian liquid, Bingham plastic (solid) and Schaeffer solid (powder in the quasi-static regime) compared with the proposed new constitutive equation, (7) that contains the viscous term as in equation (11-5, "modified Schaeffer law"). The torque on the inner rotating cylinder is given as a function of the rotational speed of the inner cylinder (in RPM). The Schaeffer powder (solid) yields a constant torque as a function of rotation (or shear) rate, as expected. The Bingham plastic also yields a constant torque at low shear rates but then behaves like the Newtonian fluid and, above a rotational speed of about 10 RPM, the data become indistinguishable. Two curves are presented in Figure 10 for the powder in the intermediate regime ("New model" in the figure) for a power-law index of $n=0.5$ and $n=1.5$, respectively. One can see that the viscous term has a significant influence on the modified Schaeffer law and the torque is shear rate dependent at higher shear rates as was found experimentally in Figure 8 (the shear stress and torque are related linearly as in equation (2)). 
Figure 11 shows the ratio of average shear to normal stresses on the rotating cylinder versus the shear rate with the numerical solution using the new constitutive equation (11-5) and compares calculations with experimental data in Figure $9(\mathrm{n}=0.72$ and $n=1.03$ and $b=0.13$ and $b=0.06$, respectively). Two zones can be seen, solid like behavior at low shear rate where the ratio is constant and independent of the shear rate reflecting quasi-static flow, and fluid like behavior as the shear rate increases beyond a critical value where the ratio is dependent on shear rate. This result in itself does not demonstrate that the model correctly predicts the experimental data since the input to the model was the experimental data itself (with a slight modification using tan $\phi$ instead of the constant coefficient "a" as mentioned above). It shows however that the simplifications introduced by the continuum approach and the numerical method used do not introduce excessive errors. The advantage of the numerical solution is that velocity, stress distributions and overall torque can be calculated for different geometries, and some examples are given below.

Figure 12 gives the torque on a slice of the Couette of height "unity" for all four continua as a function of the rotational speed of the inner cylinder (in RPM). Different simulations were performed for the concentric cylinder (taken partially from Figure 10) and for the case when the inner cylinder is moved off-center by one quarter of the gap ( 0.25 inches) and half of the size of the shear gap ( 0.5 inches). An important conclusion from these simulations is that the torque is not influenced in a significant way by the eccentricity for any continua considered herein. Of course, this is true only as long as the eccentricity is kept to less or equal to half of the shearing gap as shown in the figure. This conclusion is relevant from an experimental point of view since small eccentricities and "wobbles" of rotating parts are very difficult to avoid in practice. Note that the torque generated by the Schaeffer solid is constant for all shear rates since the pressure is constant (constant stress experiment); this is to be expected for a solid powder moving in the quasi-static regime. The appropriate change in solid fraction (or bulk density) to achieve this is not captured by the model since it is assumed that the powder is incompressible. Experiments performed with an off-center cylinder (situated 0.25 inches off center) actually proved that there is no difference in the behavior of the powder and the overall torque is practically the same.

Figure 13 gives similar results for the four continua for the concentric case where an additional stationary cylinder is introduced in the middle of the gap. This cylinder is denoted "obstacle" in the figure and occupies a quarter of the gap (at 0.25) and half of the gap (at 0.5). The Newtonian fluid and the Bingham plastic with the same viscosity appear to be quite sensitive to the presence of the obstacle, as expected, while the Schaeffer solid seems to be quite insensitive. This last result is again to be expected since previous studies showed (Tardos et al., 2003) that the shearing zone in the quasi-static regime is actually very close to the moving surface and decreases exponentially from the boundary. For this reason, the presence of an obstacle in the middle of the shearing gap, even if it occupies one half of the distance, does not affect the torque on the rotating cylinder to a significant degree. The behavior of the modified Schaeffer solid i.e., using the constitutive law in equation (11-5), is intermediate between the viscosity containing continua and the Schaeffer solid. The larger obstacle generates a somewhat higher torque but experiments performed so far with a similar system (in which the "obstacle" occupies half the shear gap) yielded results that are inconclusive in the sense that the fluctuation of the experimental torque are larger then the difference in torque between the absence and presence of the obstacle.

Figure 14 is an attempt to show the complex capabilities of the numerical model. An exhaustive calculation of all the variables at all spatial positions is beyond the scope of the 
present work. The figure contains the local rotational speed (in RPM), the shear rate $\left(\mathrm{sec}^{-1}\right)$, pressure, viscosity and stress components using a color scheme and the geometry of the system for "Crushed glass" employing data from Figure 13 at a rotation rate of 10 RPM. The different quantities are given as a function of bed height which is zero at the bottom and increases in the vertical direction upwards to a maximum value of unity. The white point on the bottom plane is the center location of the "obstacle". "No slip" boundary conditions are assumed on both cylinders (rotating, inner and stationary, outer) as well as on the bottom plate that is assumed to be stationary. The different quantities are calculated in the figure at a radial distance of $1 / 3$ into the shearing zone $(R=\sim 2.32$ inches). One can see the spatial distribution of the physical quantities in this more complex geometry: velocity, shear rate, normal stress and shear stress ( $T_{11}$ and $T_{22}$ are the normal stresses in the " 1 or rr" and " 2 or $\theta \theta$ " directions and $T_{12}$, is the shear stress). The velocity and shear rate are linearly dependent and were used to check the response of the constitutive law with respect to the shear rate. The jump of the normal stress in the presence of the obstacle in the gap is clearly reflected on the first $\left(T_{11}\right)$ and second component $\left(T_{22}\right)$ as well as in the shear and wall stresses. Moreover, wall shear stresses may have an important influence on the material flow-properties, as for instance the formation of granular chains. Another important conclusion (see in particular the three quantities calculated on the top row) is that the system becomes height $(z)$ independent at approximately $2 / 3$ away from the (stationary) bottom or approximately at a distance equal to the width if the shear gap.

\section{Conclusions}

We study in this work experimentally and numerically the flow of dry, frictional powders in the quasi-static and intermediate regimes using the simple geometry of the Couette device. We measure normal and shear stresses on the shearing surface and propose a constitutive equation valid in regimes. This constitutive equation is then used in a new, specially developed numerical scheme realized in the FEM package FeatFlow to solve the continuum equations of motion and to obtain stress and velocity distributions in the powder. While the measurements to obtain the constitutive equation are performed in a concentric Couette device, the numerical scheme is used to predict the torque and stresses in two additional geometries: an eccentric Couette device where the inner, rotating cylinder is placed offcenter with different eccentricities and a more complicated geometry where a cylindrical body is introduced in the middle between the rotating and stationary cylinders and obstructs part of the shearing gap.

We find that it is possible to measure normal stresses on the shearing wall of the Couette device inside the granular layer and calculate the ratio of the average shear to normal stress as a function of shear rate. It appears that the dynamic angle of friction of the powder is reproduced by this ratio only at very low shear rates. As the shearing rate increases, the ratio of the stresses also increases due to collisions between particles that sustain some load. We show that a modified Couette device with slow axial flow superimposed on the shearing motion can be used to determine the constants ("b" and " $n$ ") of a yield condition for any material that is somewhat free flowing and thus can be used as a "powder rheometer". The yield condition is valid in both the quasi-static as well as the "intermediate" regimes of flow and contains a term characterizing "solid'-like behavior and an additional term that captures some "fluid"-like properties at higher shear rates.

The paper describes a new finite element solver FeatFlow of the generalized Navier-Stokes equations that uses, in addition to the yield condition determined experimentally for a 
relatively free flowing powder, a generalized viscosity that describes a Newtonian fluid, a Bingham Plastic, an incompressible frictional powder (Schaeffer solid) and a power-law fluid. We use the numerical method to validate some experimental measurements and calculate the torque in the Couette device in three different geometries: a concentric, two cylinder, arrangement and two new geometries in which the cylinder is positioned eccentric in the Couette and one where an additional cylindrical object is placed into the shearing gap and obstructs parts of it.

Finally, we show in Figure 14 some of the capabilities of the model to predict a wide variety of parameters for the most complicated geometry that of the "obstacle" occupying $1 / 2$ of the shearing zone. We show, for example, that the different quantities predicted by the model become height independent at a distance roughly equal to the width of the shear gap. While the model predicts all stresses in the shear zone and the obstacle, experiments to check the torque on the rotating cylinder where inconclusive since the fluctuations of the torque were about equal in magnitude with the predicted difference in torque with and without the obstacle. Further work in measuring the local stresses and forces on the obstacle are in progress to further check the predictions of the model.

Acknowledgement. This work was supported by the German Research Association (DFG) through the collaborative research centre SFB/TRR 30 and through the grants TU 102/21-1. Additional support from the US Department of Energy under grant \#RF-75740-00-01 and a Geleese fellowship from the City University of New York Graduate Centre to MKL, are greatly appreciated. Extensive discussions with Drs. Paul Mort of Procter\&Gamble Corporation and James N. Michaels from Merck and Co. Inc regarding the subject matter of this work are also acknowledged as is a stipend for MKL from the P\&G Company to support this work. 


\section{References}

1. Campbell, C.S., "Granular material flows - an overview", Powder Technology, Vol. 162, pp. 208229, (2006).

2. Bulícek, M. and Kaplický, P., Incompressible fluids with shear rate and pressure dependent viscosity: regularity of steady planar flows, Discrete and continuous dynamical systems series S, 1 (Vol. 1, pp. 41-50 (2008)

3. Bulícek, M., Malék, J. and Prazák, D., On the dimension of the attractor for a class of fluids with pressure dependent viscosities, Communications on pure and applied analysis, Vol. 4, pp. 805822, (2005)

4. DaCruz, F., Emam, S., Prochnow, M., Roux, JN., Chevoir, F., 'Rheophysics of dense granular materials: discrete simulation of plane shear flows', Phys. Rev. E. 72, 021309, (2005).

5. Hron, J., Malek, J., Necas, J. and Rajagopal, K. R., Numerical simulations and global existence of solutions of two-dimensional flows of fluids with pressure- and shear-dependent viscosities, Mathematics and Computers in Simulation, Vol. 61, pp. 297-315, (2003)

6. Jenike, A.W., Storage and flow of solids, Bulletin of University of Utah, Bulletin No. 123, Vol. 53, No.26, (1964).

7. Jackson, R., Some features of the granular materials and aerated granular materials, J. of Rheology, Vol. 30 (5), pp. 907-930, (1986).

8. Kuzmin, D., Löhner, R. and Turek, S., Flax-Correction Transport: Principles, Alghorithms, and Applications. Springer (2005)

9. Luding, S. Shear flow modeling of cohesive and frictional powders, Powder Technology, Vol. 158, pp. 45-50, (2005).

10. Malék, J. Necãs, J. and Rajagopal, K. R., Global Existence of Solutions for Flows of Fluids with Pressure and Shear Dependent Viscosities Applied Mathematics Letters, Vol. 15 pp. 961-967, (2002)

11. Moreno-Atanasio, R., Anthony, S.J. and Ghadiri, M., Analysis of flowability of cohesive powders using Discrete Element Methods, Powder Technology, Vol. 158, \# 1-3, pp. 41-57, (2005).

12. Mort P.R., Tardos, G.I. and Talu, I., "Characterizing the transition between the quasi-static and intermediate regimes in weakly cohesive granular flows", paper presented at the Annual AICHE meeting, San Francisco, November (2003).

13. Muguruma, Y., Tanaka, T. and Tsuji, Y., Numerical simulation of particulate flow with liquid bridge between particles, Powder technology, vol. 109, (2000).

14. Nedderman, R. M., 'Statics and Kinematics of Granular Materials', Cambridge University Press, (1992).

15. Ouazzi, A., "Finite element simulation of non-linear fluids: Application to granular materials and powders", PhD dissertation, Department of Applied mathematics, University of Dortmund, Germany, Shaker Verlag, Aachen, Germany, (2006).

16. Ouazzi, A. and Turek, S., Numerical Methods and Simulations Techniques for Flow with Shear and Pressure dependent Viscosity, Feistauer, M Dolejsi, V.; Knobloch, P.; Najzar, K, pp. 668676, Numerical Mathematics and Advanced Applications, Springer, Enumath 2003, Prague, ISBN. 3-54021460-1, (2003)

17. Ouazzi, A., Turek S. and Hron, J., "Finite element methods for the simulation of incompressible powder flows", Communications in Numerical methods in Engineering, Vol. 21, pp. 581-596, (2005).

18. Prasad, S. and Rajagopal, K. R., Flow of a fluid with pressure dependent viscosity due to a boundary that is being stretched, J. Applied mathematics and computation, Vol. 173, pp. 50-68, (2006)

19. Rannacher, R. and Turek, S., A simple nonconforming quadrilateral Stokes element, Numer. Meth. Par. Diff. Eq., Vol 8, pp. 97-111, (1992)

20. Renardy, M., Parallel shear flows of fluids with a pressure-dependent viscosity, Journal of nonnewtonian fluid mechanics, Vol. 114 (2-3) 229-236, (2003)

21. Savage, S.B., Analyses of slow high-concentration flows of granular materials, J. Fluid Mechanics, Vol. 377, pp. 1-26, (1998) 
22. Savage, S.B. and Sayed, M., Stresses developed by dry cohesionless granular materials sheared in an annular shear cell, J Fluid Mechanics, Vol. 142, pp. 391-430, (1984).

23. Schaeffer, D.G., Instability in the evolution equations describing incompressible granular flow, J. of Differential Equations, Vol. 66, 19-50, (1987).

24. Schae er, D. G., Mathematical issues in the continuum formulation of slow granular flow, In: D.G. Joseph and D.G. Schae er, Editors, Two Phase Flows and Waves, Springer Verlag, 118129, (1990).

25. Srivastava, A. and Sundaresan, S., "Analysis of a frictional kinetic model for gas-particle flow", Powder Technology, Vol. 129, pp.72-85, (2003).

26. Tardos, G.I., A fluid mechanics approach to slow, frictional powder flows, Powder Technology, Vol. 92, pp. 61-74, (1997)

27. Tardos, G.I., Khan, M.I. and Schaeffer, D.G., Forces on a slowly rotating, rough cylinder in a Couette device containing a dry, frictional powder, Physics of Fluids, 10 (2), pp. 335-341, (1998).

28. Tardos G.I., McNamara, S and Talu, I., "Slow and Intermediate flow of a frictional bulk powder in the Couette geometry", Vol. 131, pp. 23-39, Powder Technology, (2003).

29. Tardos G.I. and Mort, P.J. "Dry Powder Flows", Chapter 9 in "Multiphase Flow Handbook", C. Crowe, (Ed.), CRC Press, Boca Raton, Florida, October (2005).

30. Turek, S. and Ouazzi, A., Unified edge-oriented stabilization of nonconforming FEM for incompressible flow problems: Numerical investigations, J. Numer. Math., Vol. 15, pp. 299-322, (2007)

31. Turek, S., Ouazzi A. and Schmachtel, R., "Multi-grid methods for stabilized nonconforming finite elements for incompressible flow involving the deformation tensor formulation", J. Numer. Math., vol. 10, pp. 235-248, (2002).

32. Turek, S., Efficient solvers for incompressible flow problems: An algorithmic computational approach, Springer, Vol. 6, LNCSE, (1998). 


\section{Appendix - Detailed description of the numerical methods in FeatFlow}

Let us consider the flow of the stationary, generalized Navier-Stokes problem in equation (10) in a bounded domain $\Omega \subset R^{2}$. The weak formulation: find $(u, p) \in X \times M$ such that

$$
\begin{array}{r}
\int_{\Omega} 2 \mu\left(\gamma_{I I}, p\right) \dot{\gamma}(u): \dot{\gamma}(v) d x+\int_{\Omega}(u \cdot \nabla u) v d x \\
+\int_{\Omega} p \nabla \cdot d x=\int_{\Omega} f v d x, \quad \forall v \in X ; \\
\int_{\Omega} q \nabla \cdot v d x=0, \quad \forall q \in M .
\end{array}
$$

with $X=\left(H_{0}^{1}(\Omega)\right)^{N}, M=L^{2}(\Omega)$ for the Newtonian case.

We consider a subdivision $K \in T_{h}$ consisting of quadrilaterals in the domain, and we employ the nonconforming Rannacher-Turek element (Rannacher and Turek (1992)). For any quadrilateral let denote $(\xi, \eta)$ a local coordinate system obtained by joining the midpoints of the opposing faces of the element $K$, Then, in the nonparametric case, we set on each element

$$
\ddot{Q}_{1}(K):=\left\{1, \xi, \eta, \xi^{2}-\eta^{2}\right\}
$$

The degrees of freedom are determined by the nodal functions

$$
\begin{gathered}
\left\{F_{\Gamma}^{(a, b)}(\cdot), \Gamma \subset \partial T_{h}\right\}, \\
F_{\Gamma}^{\alpha}=|\Gamma|^{-1} \int_{\Gamma} v d s \quad \text { or } \quad F_{\Gamma}^{\alpha}:=v\left(m_{\Gamma}\right), \quad\left(m_{\Gamma} \text { midpoint of the edge } \Gamma\right)
\end{gathered}
$$

such that the finite element space can be written as

$$
W_{h}^{a, b}:=\left\{\begin{array}{r}
v \in L_{2}\left(\Omega_{h}\right), v \in \ddot{Q}_{1}, \forall K \in T_{h}, v \text { continuous w.r.t. all } \\
\text { nodal funtionals } F_{\Gamma_{i j}(\cdot)}^{a, b}, \text { and } F_{\Gamma_{i 0}}^{a, b}(v)=0, \forall \Gamma_{i 0}
\end{array}\right\} ;
$$

Here, $\Gamma_{i, j}$ denote all inner edges sharing the two elements $i$ and $j$, while $\Gamma_{i 0}$ denote the boundary edges of $\partial \Omega_{h}$. In this paper, we always employ version 'a' with the integral mean values as degrees of freedom. Then, the corresponding discrete functions will be approximated in the spaces

$$
V_{h}:=W_{h}^{a} \times W_{h}^{a}, L_{h}:=\left\{q_{h} \in L_{2}(\Omega), q_{h \mid K}=\text { const. } \forall K \in T_{h}\right\} .
$$

There are well-known situations for standard FEM methods when severe numerical problems may arise, namely in the case of convection dominated problems. Then, numerical difficulties arise for instance for medium and high Re numbers since the standard Galerkin formulation usually fails and may lead to numerical oscillations and to convergence problems of the iterative solvers. Among the stabilization methods existing in the literature for these types of problems, we use the proposed one in Ouazzi (2006) and Turek and Ouazzi (2007) which is based on the penalization of the gradient jumps over element boundaries. In 2D, the additional stabilization term $\mathrm{J} \mathrm{u}$, acting only on the velocity $\mathrm{u}$ in the momentum equations, takes the following form (with $h_{E}=|E|$ )

$$
\langle J u, v\rangle=\sum_{\text {edge } \mathrm{E}} \max \left(\delta \mu h_{E}, \delta^{*} h_{E}^{2}\right) \int_{E}[\nabla u]:[\nabla v] d s
$$


which can be simply added to the original bilinear form. Summarizing, in the underlying test cases which require the solution of stationary problems, efficient Newton-type and multigrid solvers can be easily applied for such highly accurate stabilization techniques (see, Kuzmin et al. (2005) for more details) which are the basis of the subsequent numerical analysis.

The nonlinearity is first handled on the continuous level. Let $u^{l}$ be the initial state, the (continuous) Newton method consists of finding such that $u \in V$

$$
\begin{array}{r}
\int_{\Omega} 2 \mu\left(\gamma_{\Pi}\left(u^{l}\right), p^{l}\right) \dot{\gamma}(u): \dot{\gamma}(v) d x \\
+\int_{\Omega} 2 \partial_{1} \mu\left(\gamma_{\Pi}\left(u^{l}\right), p^{l}\right)\left(\dot{\gamma}^{l}\left(u^{l}\right): \dot{\gamma}(u)\right)\left(\dot{\gamma}\left(u^{l}\right): \dot{\gamma}(v)\right) d x \\
+\int_{\Omega} 2 \partial_{2 \mu}\left(\gamma_{\Pi}\left(u^{l}\right), p^{l}\right)\left(\dot{\gamma}\left(u^{l}\right): \dot{\gamma}(v)\right) p d x \\
=\int_{\Omega} f v d x-\int_{\Omega} 2 \mu\left(\gamma_{\Pi}\left(u^{l}\right), p^{l}\right) \dot{\gamma}\left(u^{l}\right): \dot{\gamma}(v) d x \quad \forall v \in V,
\end{array}
$$

where $\partial_{i}(.,.) ; i=1,2$ is the partial derivative of $\mu$ related to the first and second variables respectively; for more details, see Ouazzi. and Turek (2003).

The resulting auxiliary sub-problems in each Newton step consist of $(u, p) \in X \times M$ finding as solutions of the linear (discretized) systems

$$
\left\{\begin{aligned}
A\left(u^{l}, p^{l}\right) u+\delta_{d} A^{*}\left(u^{l}, p^{l}\right) u+B p+\delta_{p} B^{*}\left(u^{l}, p^{l}\right) p & =R_{u}\left(u^{l}, p^{l}\right) \\
B^{\mathrm{T}} u & =R_{p}\left(u^{l}, p^{l}\right) .
\end{aligned}\right.
$$

where $R_{u}(.,$.$) and R_{p}(.,$.$) denote the corresponding nonlinear residual terms for the$ Momentum and continuity equations, and the operators $A\left(u^{l}, p^{l}\right), B\left(u^{l}, p^{l}\right), A^{*}\left(u^{l}, p^{l}\right)$, and $B^{*}\left(u^{l}, p^{l}\right)$ are defined as follows:

$$
\begin{gathered}
\left\langle A\left(u^{l}, p^{l}\right) u, v\right\rangle=\int_{\Omega} 2 \mu\left(\gamma_{\Pi}\left(u^{l}\right), p^{l}\right) \dot{\gamma}(u): \dot{\gamma}(v) d x \\
\left\langle A^{*}\left(u^{l}, p^{l}\right) u, v\right\rangle=\int_{\Omega} 2 \partial_{1} \mu\left(\gamma_{\Pi}\left(u^{l}\right), p^{l}\right)\left(\dot{\gamma}\left(u^{l}\right): \dot{\gamma}(u)\right)\left(\dot{\gamma}\left(u^{l}\right): \dot{\gamma}(v)\right) d x \\
\langle B p, v\rangle=\int_{\Omega} p \operatorname{div} v d x \\
\left\langle B^{*}\left(u^{l}, p^{l}\right) p, v\right\rangle=\int_{\Omega} 2 \partial_{2} \mu\left(\gamma_{\mathbb{I I}}\left(u^{l}\right), p^{l}\right)\left(\dot{\gamma}\left(u^{l}\right): \dot{\gamma}(v)\right) p d x
\end{gathered}
$$

Finally, the following part is devoted to give a brief description of the involved solution techniques for the resulting linear systems. For the nonconforming $\tilde{Q}_{1} / Q_{0}$ Stokes element a 'local pressure Schur complement' preconditioner (see Turek (1998)) as generalization of the so-called ' $V$ anka smoothers' is constructed on patches $\Omega_{i}$ which are ensembles of one single or several mesh cells, and this local preconditioner is embedded as global smoother into an outer block Jacobi/Gauß-Seidel iteration which acts directly on the coupled systems of generalized Stokes, resp., Oseen type as described in Turek et al. (2002). If we denote by $R_{u}$ and $R_{p}$ the discrete residuals for the momentum and continuity equation which include the complete stabilisation term due to the modified bilinear form as described in (19), one smoothing step in defect-correction notation can be described as 


$$
\begin{aligned}
{\left[\begin{array}{l}
u^{l+1} \\
p^{l+1}
\end{array}\right] } & =\left[\begin{array}{l}
u^{l} \\
p^{l}
\end{array}\right] \\
& +\omega^{l} \sum_{i}\left(\begin{array}{cc}
\left(\tilde{A}+\delta_{d} \tilde{A}^{*}+J\right)_{/ \Omega_{i}} & \tilde{B}_{/ \Omega_{i}}+\delta_{p} B_{/ \Omega_{i}}^{*} \\
\tilde{B}_{/ \Omega_{i}}^{\mathrm{T}} & 0
\end{array}\right)^{-1}\left[\begin{array}{c}
\tilde{R}_{u}\left(u^{l}, p^{l}\right) \\
\tilde{R}_{p}\left(u^{l}, p^{l}\right)
\end{array}\right]
\end{aligned}
$$

with matrix $\tilde{A}, \tilde{B}, A^{*}$, and $B^{*}$ are the discrete matrices corresponding to the operators in (A9), (A10), (A11) and (A12). All components in the multigrid approach, that means intergrid transfer, coarse grid correction and coarse grid solver, are the standard ones and are based on the underlying hierarchical mesh hierarchy and the properties of the nonconforming finite elements (see Turek (1998) and Turek et al. (2002) for details). 
a.

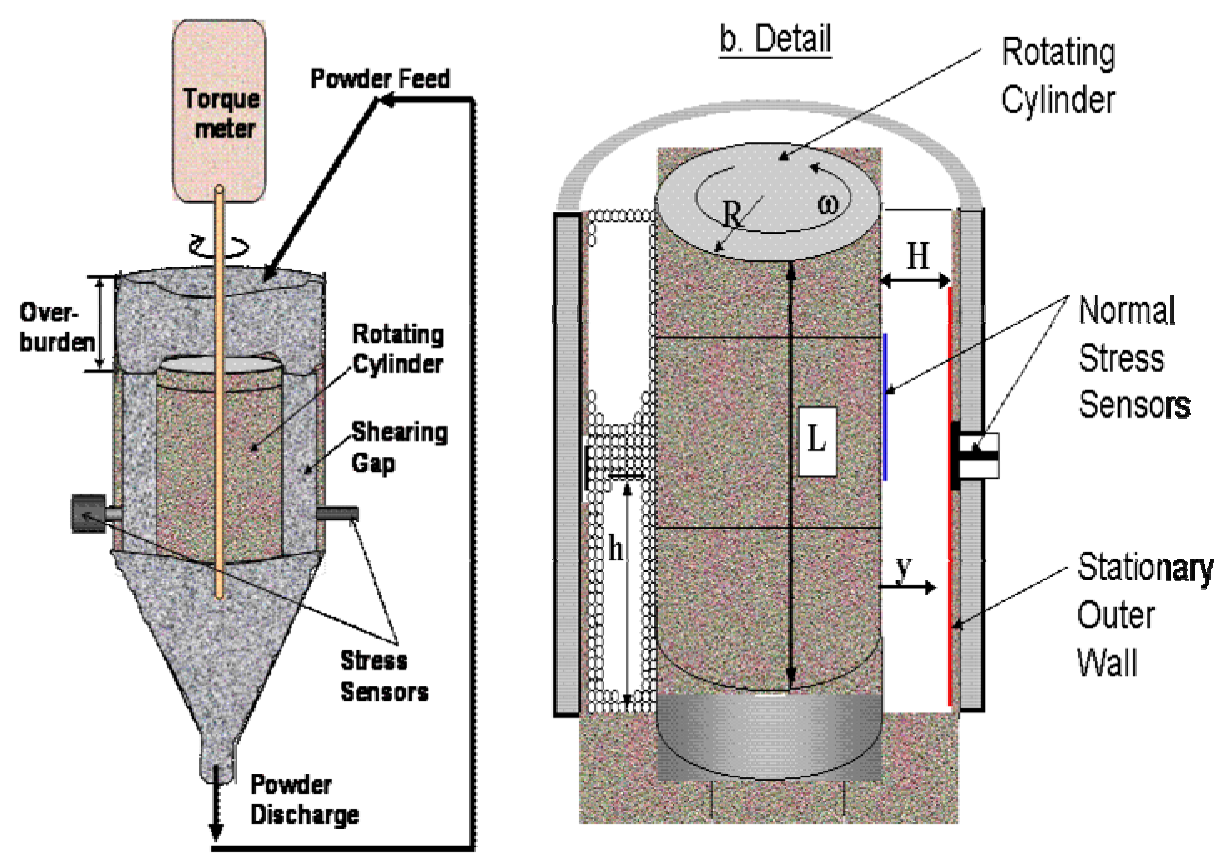

Figure 1. Schematic representation of the axial-flow Couette device (a) and detail of the stress sensors (b)

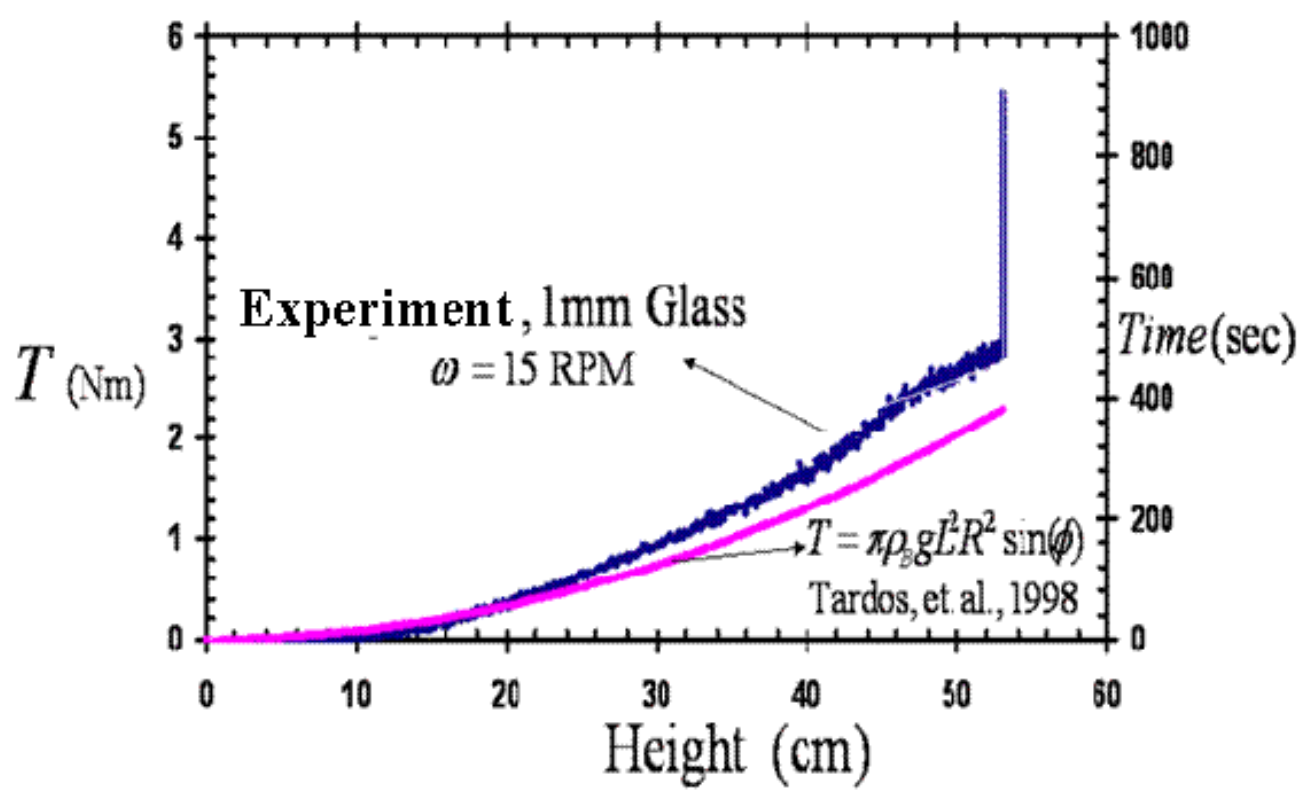

Figure 2. Filling curve: torque vs. height for $1 \mathrm{~mm}$ glass beads 

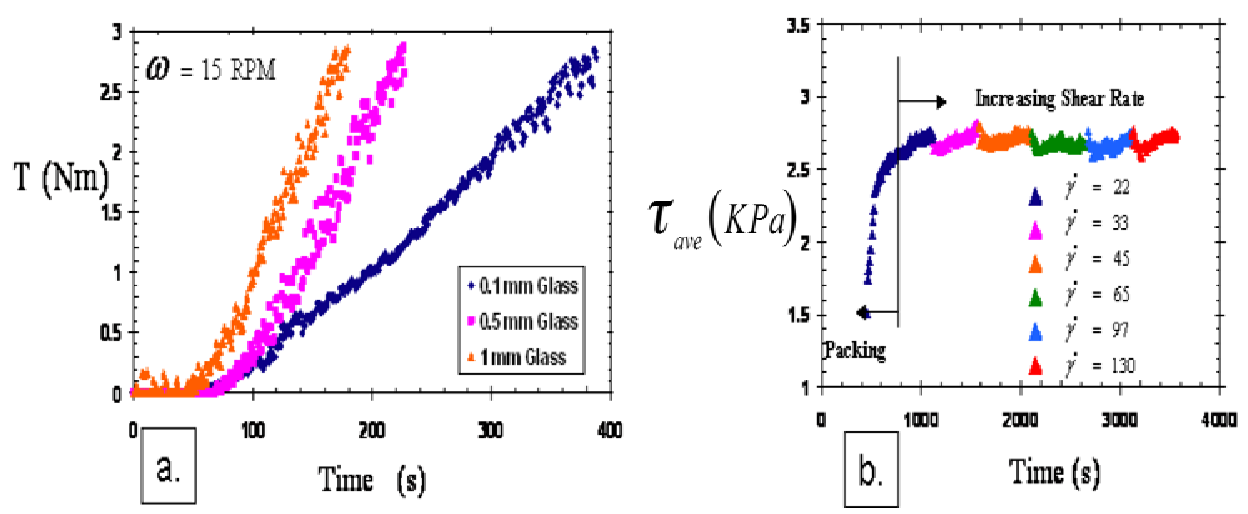

Figure 3. Batch experiments in the (no-flow) Couette device: a. torque vs. time for different particles; $b$. shear stress vs. time at different shear rates

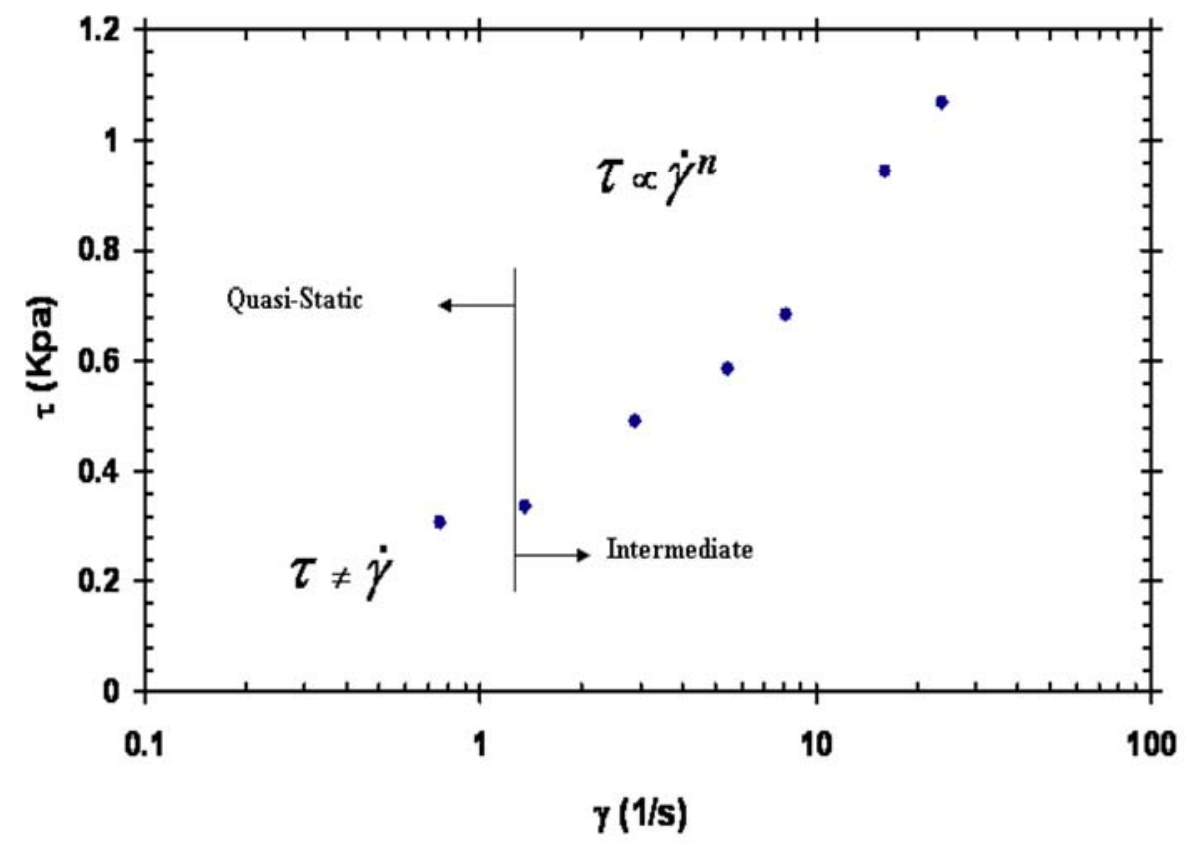

Figure 4. Axial flow experiment in the Couette device: glass particles $0.1 \mathrm{~mm}$ in diameter. 


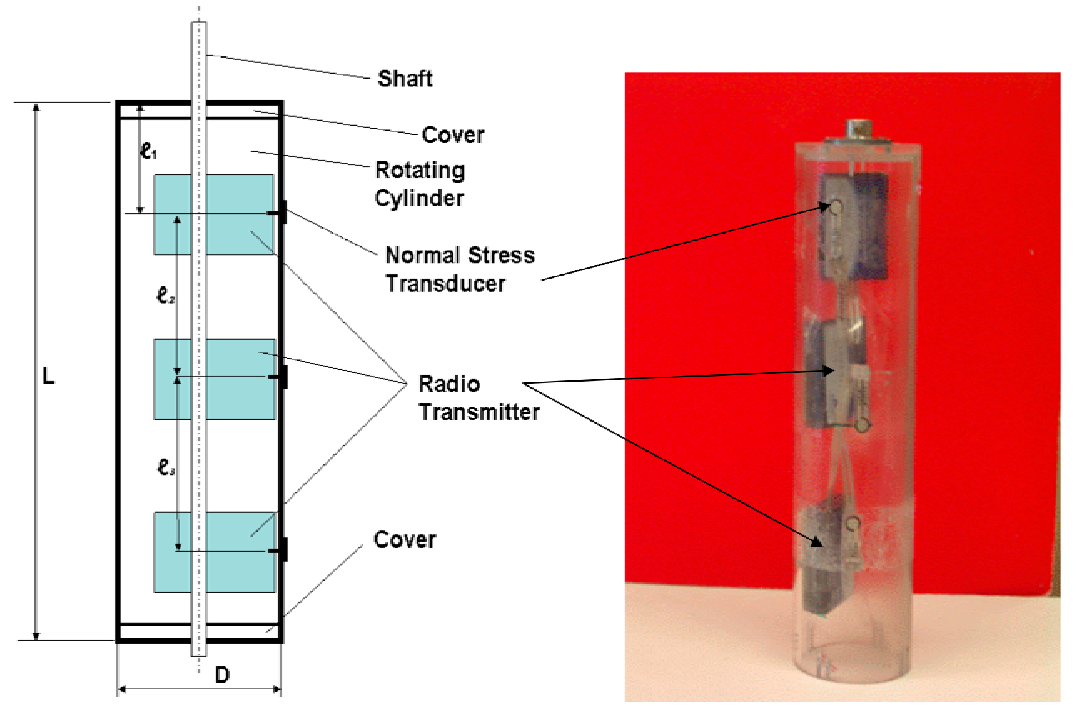

Figure 6. Schematic and picture of the Couette device with three remote normal-stress sensors.

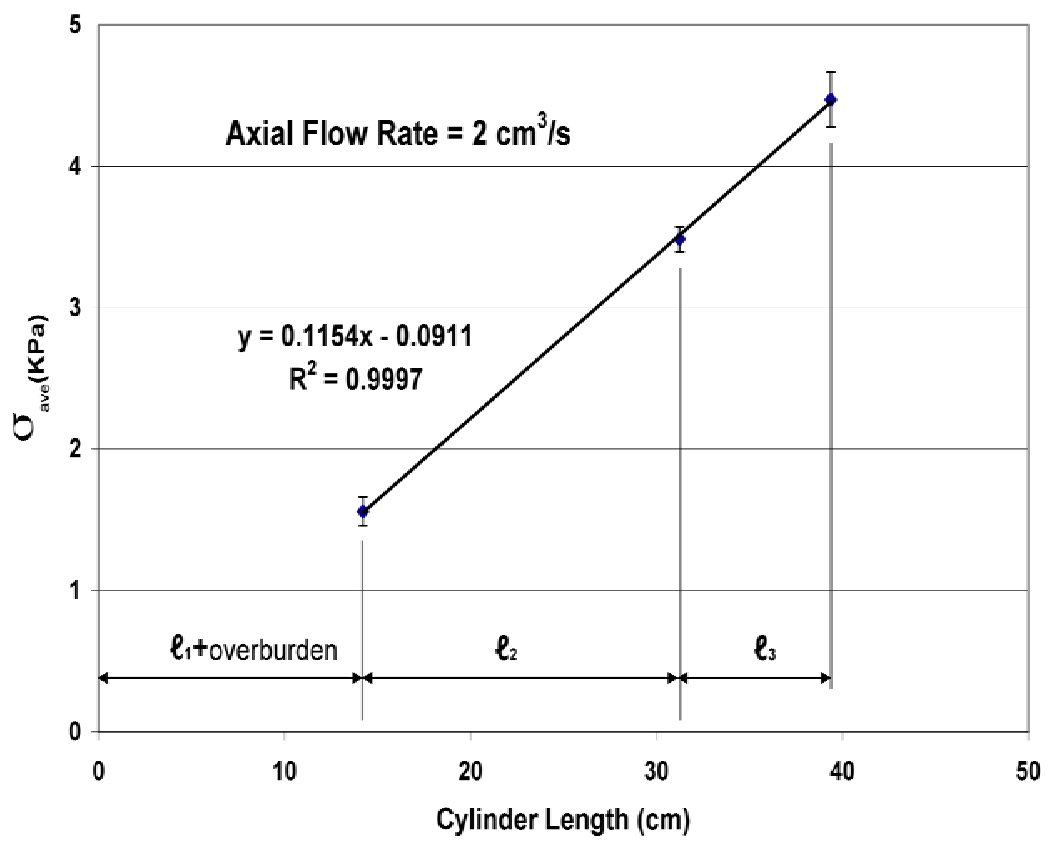

Figure 7. Normal stress as a function of height as measured on the shearing cylinder surface by remote sensing. 


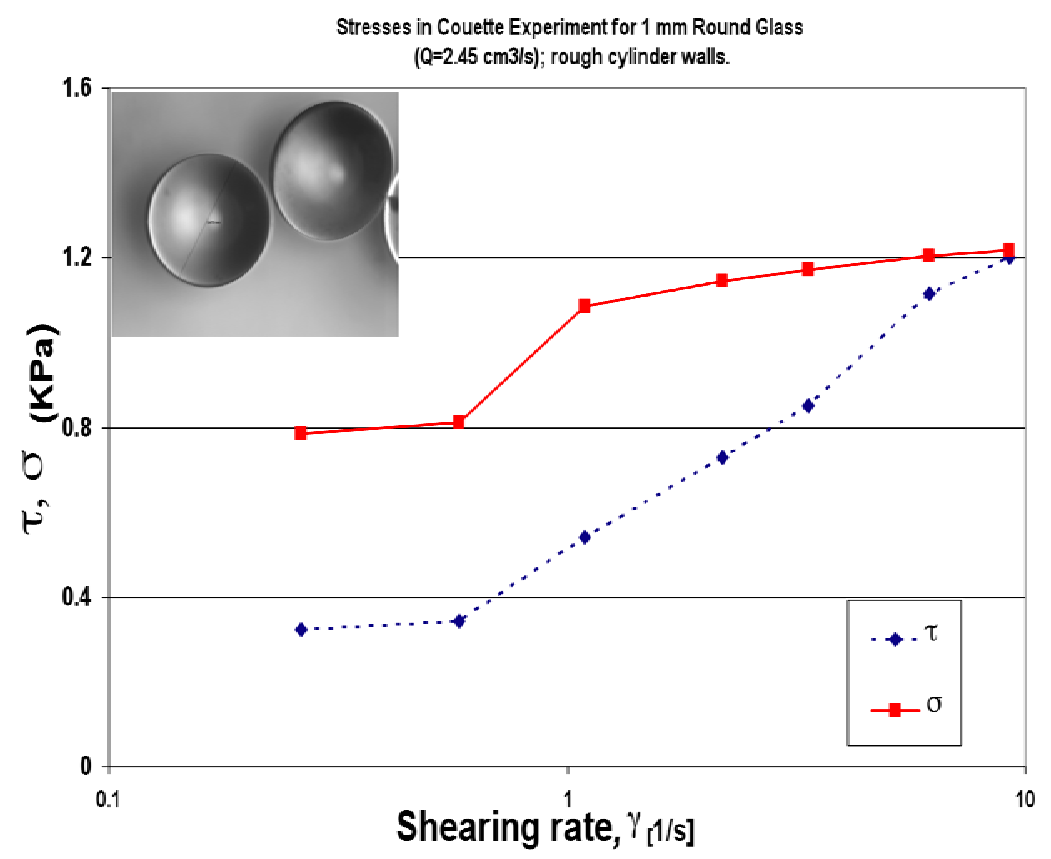

Figure 8.: Shear and Normal stress variation as a function of shearing rate in the Couette device; $1 \mathrm{~mm}$ in diameter glass particles. Insert: picture of particles

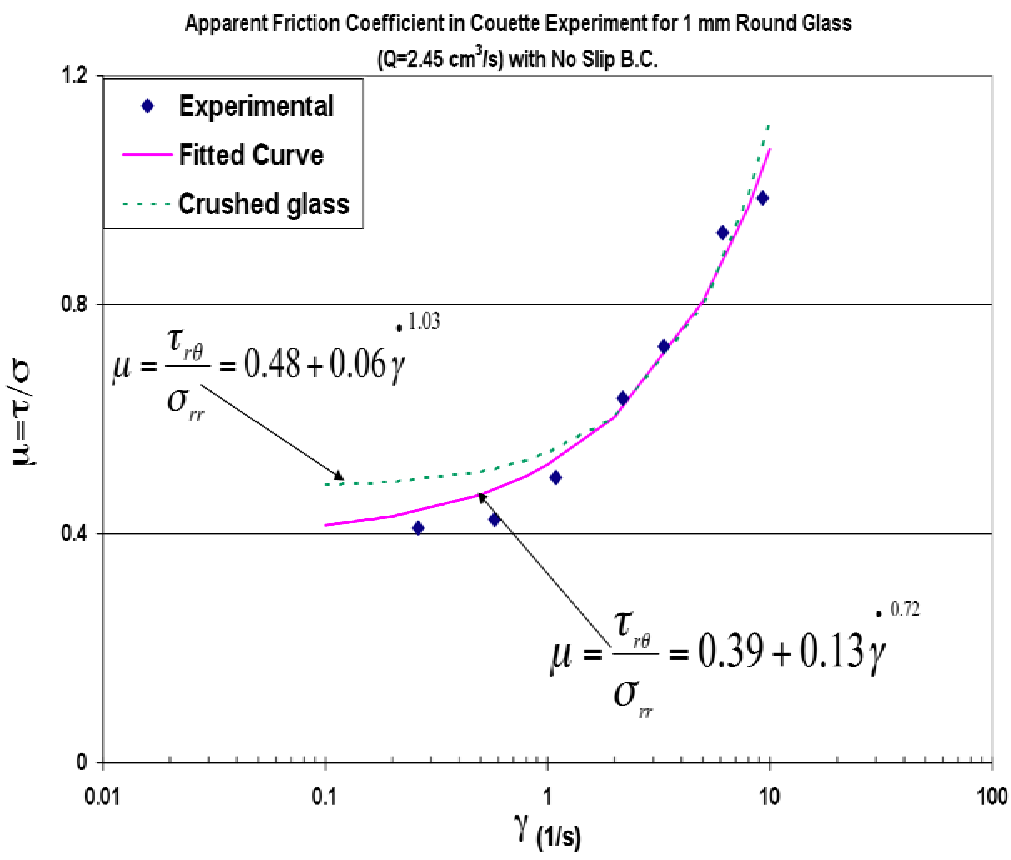

Figure 9.: Ratio of Shear to Normal stresses as a function of shearing rate (apparent friction coefficient); $1 \mathrm{~mm}$ in diameter, spherical glass particles. Dotted curve: crushed, odd shaped glass chips of $1 \mathrm{~mm}$ in size. 


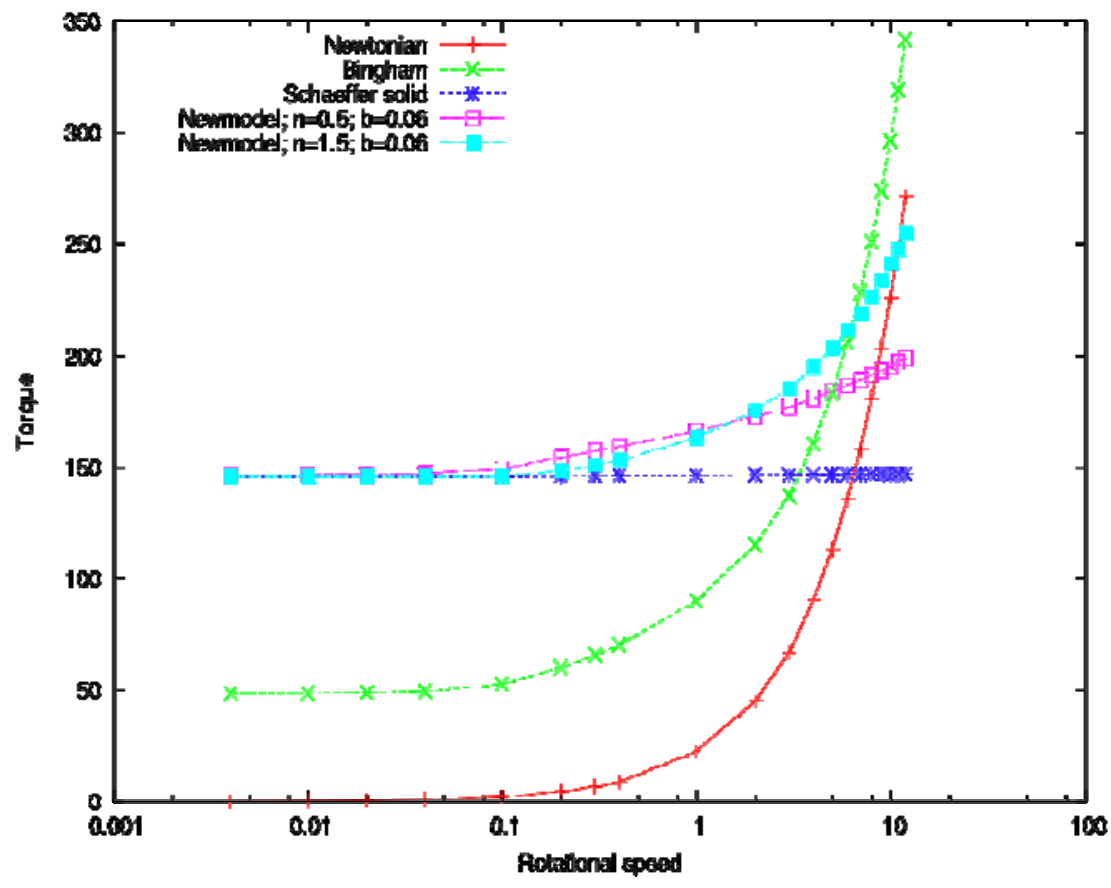

Figure 10. Numerical solution of the generalized Navier-Stokes equations for concentric cylinders for a Newtonian Fluid, Bingham plastic, Schaeffer Solid and new constitutive equation (7) containing the Schaeffer solid and a power-law fluid.

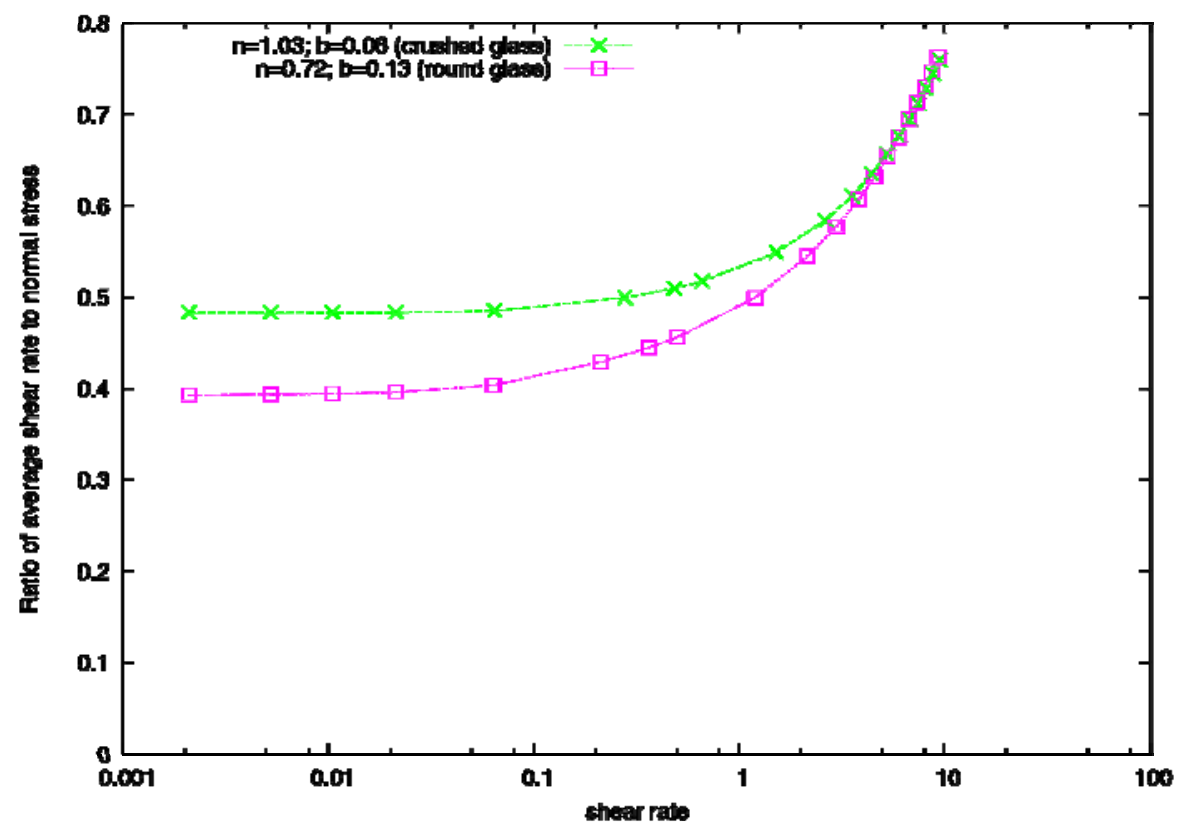

Figure 11: Numerical Calculation of ratio of average shear to normal stresses vs. shear rate using the new constitutive equation for concentric cylinders $-1 \mathrm{~mm}$ in diameter round and crushed glass. 


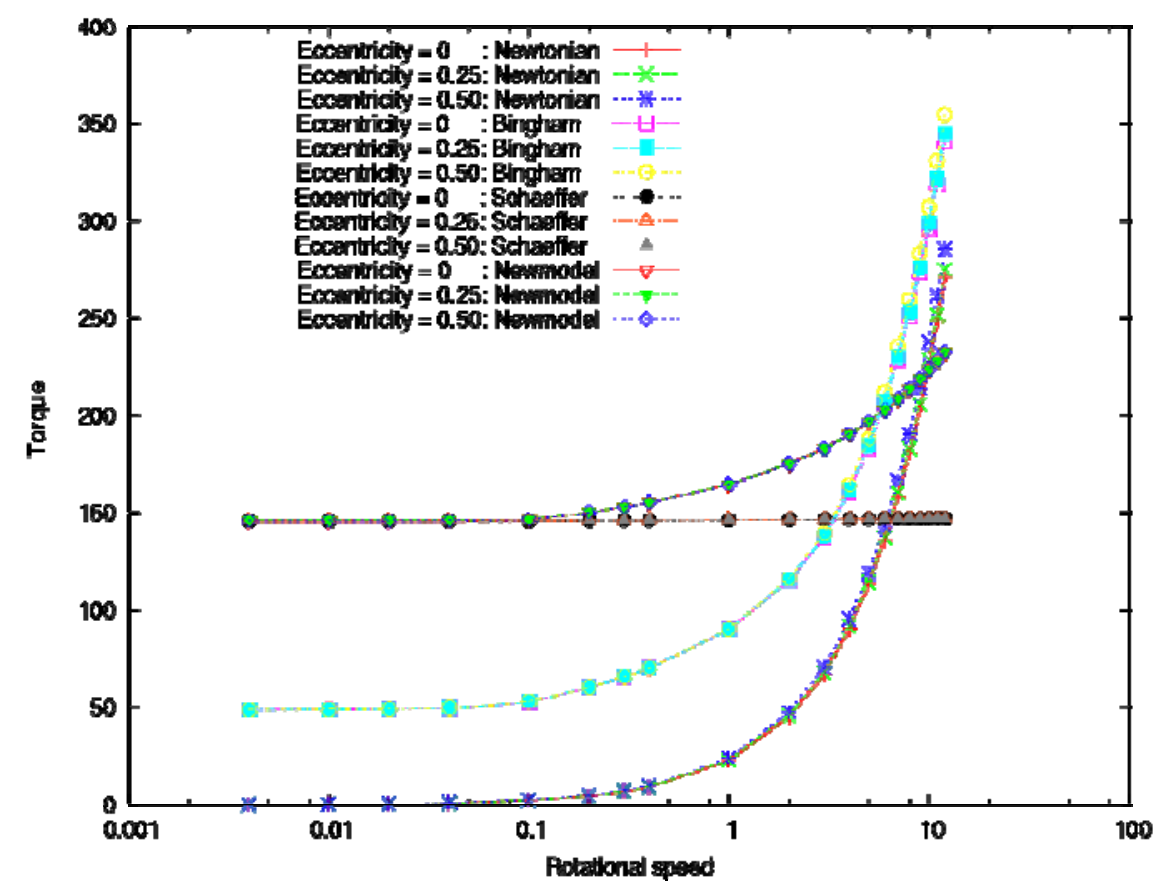

Figure 12. Numerical solution of the generalized Navier-Stokes equations for a Newtonian Fluid, Bingham plastic, Schaeffer Solid and Schaeffer solid with power-law (equation, 7). Comparison of concentric and two eccentric cylinders.

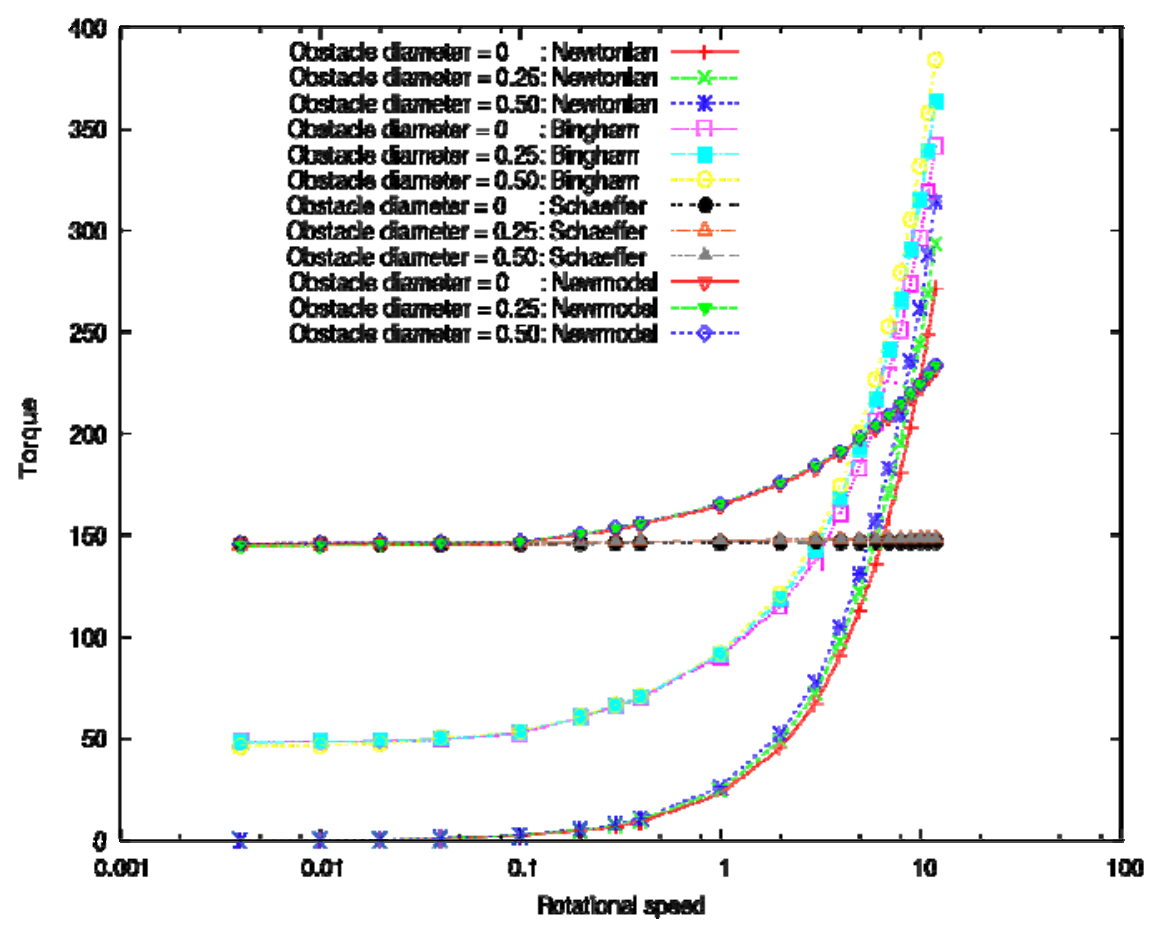

Figure 13. Numerical solution of the generalized Navier-Stokes equations for a Newtonian Fluid, Bingham plastic, Schaeffer Solid and Schaeffer solid with power-law. Concentric Couette with a cylindrical obstacle in the center of the shearing gap. 

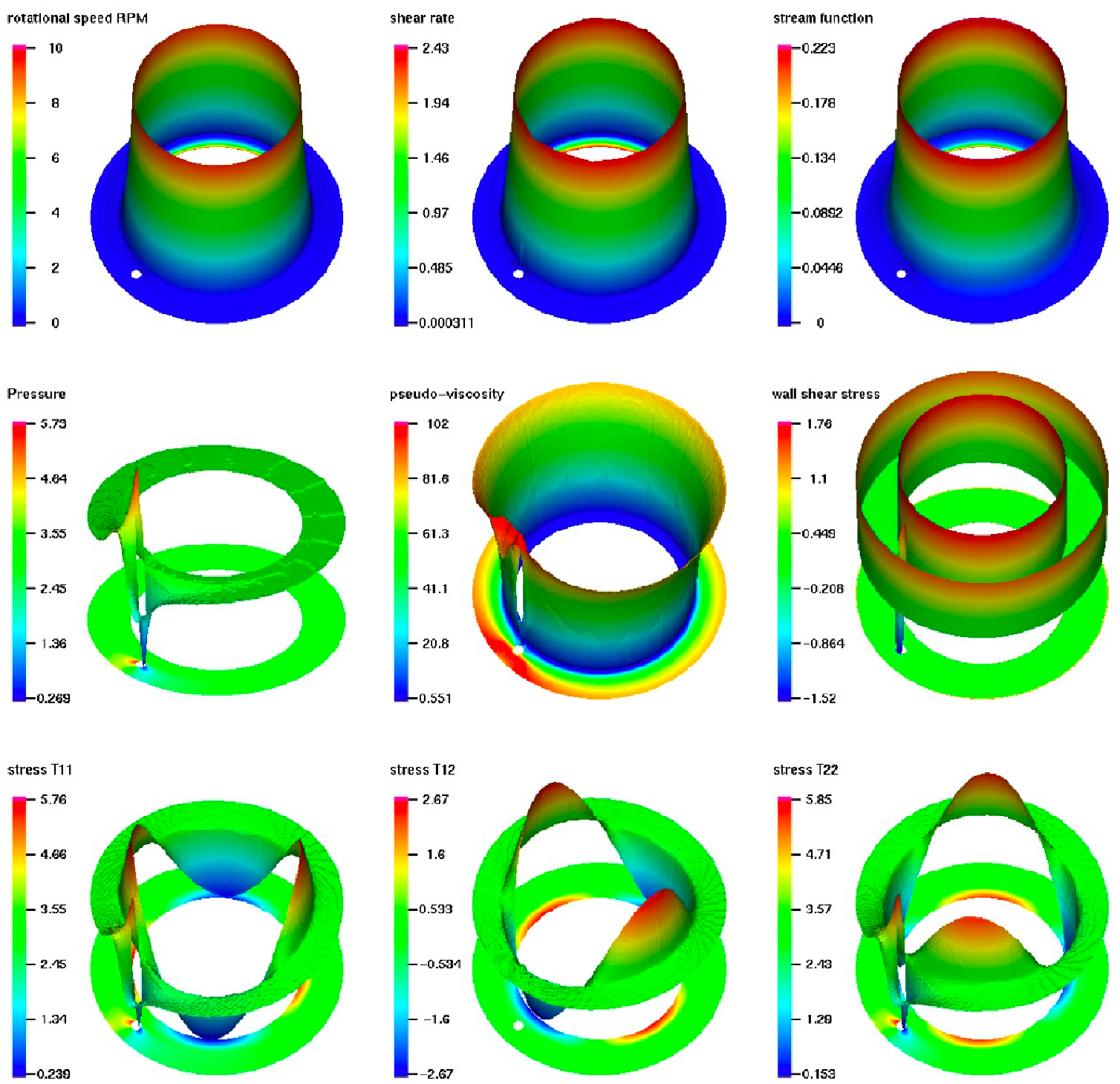

Figure 14. Numerical solution of the generalized Navier-Stokes equations for a Schaeffer solid with power-law (Equation 11-5). Concentric Couette with a cylindrical obstacle in the center occupying half the shearing gap at rotational Speed 10 RPM. 